\title{
REVIVING KNOWLEDGE ON EQUIPMENT FAILURES AND IMPROVING RISK MANAGEMENT AT INDUSTRIAL SITES
}

\author{
Paolo Bragatto \\ DIT Technological Innovations Department, Rome, Italy \\ Patrizia Agnello \\ DIT Technological Innovations Department, Rome, Italy \\ Silvia Ansaldi \\ DIT Technological Innovations Department, Rome, Italy \\ Emanuele Artenio \\ DIT Technological Innovations Department, Rome, Italy \\ Corrado Delle Site \\ DIT Technological Innovations Department, Rome, Italy
}

The knowledge about pressure equipment failure modes and rates is essential for the risk management. In the Seventies an incomparable effort was made by National Authorities, which at that time controlled the pressure equipment, aiming at providing the industry with trustable reliability parameters, which are still in us, even though repeatedly amended by experts. In order to revive and update the knowledge on this matter, the judgment of the experts is not enough and much more data on the field must be gathered. Experimental studies on a large scale are now impossible, thus the only way is to exploit potential of semantic search and the huge data in the public domain. The information scattered in the web, tied together, may provide industry and authorities with the knowledge they need to make the right decisions.

Key words: Pressure equipment, Failure Modes, Semantic Search

\section{INTRODUCTION}

The new Seveso III Directive, in force in European Union since July 2015, requires the operators to assure a safe management of equipment throughout the entire lifecycle. According to the Directive the operator of risk plants must take into account available information on best practices for monitoring and controlling, with a view to reduce the risk of system failure. The present study focuses just at the pressure equipment as critical for all industries falling in the scope of Seveso Directive, including gas, oil and chemical processing, as well as for other industries, which are out of Seveso scope, including food, textile, paper and healthcare industries. The knowledge about the pressure equipment failures is essential to manage process plants. In particular the failure rates FRs and failure modes FMs are critical to plan inspection and to safely manage the ageing of pressure plants [04]. Authorities, furthermore, use FRs for making decisions on new plants and land use planning in the framework of the Seveso legislation. Just major companies have adequate resources to manage proprietary knowledge about pressure equipment failures and update parameters about FRs and FMs, whilst minor companies, including small and medium ones, trust on public domain resources. Shared knowledge resources on equipment reliability are essential to promote dialogue between operators and authority, in a framework of transparency and equity. Unfortunately, the knowledge in the public domain is out of date. As discussed in a recent paper by Pittiglio \& al. [07], in many cases both operators and authorities are still trusting in the results of studies forty years old and more. In that age a strong command and control system was in force and ruled all safety matter, including pressure equipment, and the scholars had the chance to collect and compare data on million items in service and thousands of events, as discussed by Spencer $\mathrm{H}$. Bush [05] in a valuable reviewed published a few years later. 


\section{Recent researches}

The need of updating and sharing knowledge on this matter is recognized, but at now even most trusted sources, including FRED[12] and API 581[1], are based much more on experts' judgment than on experimental data. Nobody in recent years has continued the large studies of the Seventies, because the era of command and control is passed away and it is impossible to monitor at the whole "population" of equipment in a wide area, as the matter of pressure equipment is ruled by a much more flexible and liberal legislation, without strong central control bodies. A couple of years ago an attempt was made by exploiting open access databases [06]. Even though $\mathrm{FM}^{\mathrm{s}}$ were not included and success was partial, at now, it is the only serious attempt made by Authorities for updating shared knowledge on pressure equipment failures.

\section{OBJECTIVES}

In order to meet the needs of updating FRs and FMs parameters, it is essential to collect updated much more data on failures that occur on known population of pressure equipment. The goal of the present research is to provide credible and updated FRs and FMS and eventually revive the knowledge on pressure equipment reliability. In most European countries, including Italy, all phases of pressure systems life-cycle are driven by national regulations, but the duties are spread by a number of control bodies (both public and private). A valuable source of knowledge could be provided by collecting and exploiting the control bodies' experience. The first objective is to experiment the gathering of data at a couple of control bodies, in order to evaluate the feasibility of a larger campaign. The second objective is to evaluate the potential of the information on pressure equipment failures, spread in the web. An extensive search on the entire web is outside the scope, rather it is important matching data collected in the districts with data in public domain.

\section{EXPERIMENTAL DISTRICT}

The present study was performed with a local agency, which inspect all equipment in a small industrial district in northern Italy. Failure records were collected for 11 years, until 2013. The equipment population was 6000 items in service, including some 400 steam generators. This data has been assumed almost constant in the observation time. In the observation period there were 53 recorded events. The recorded events include both major failures with severe consequences (e.g. loss of the equipment, injuries or death, environmental damage) and minor fixable failures, which just downtime as consequence. For each event has been produced a detailed sheet, describing the type of failure, the type of equipment, the material and the age of the equipment, the affected part, the consequences, the assumed causes. Further details and photographs, as relevant, have been enclosed too. For the purpose of comparison was analyzed also a collection of recorded faults present in the public domain [11]. It contains 53 events collected in 7 years (1995 - 2001) in a highly industrialized Italian area (Emilia-Romagna), with a population of about 46,000 in equipment pressure.

\section{PHASES AND METHODS}

We divided the work in 4 phases.

Phase 1 Reports' collection. Reports about breakdowns, incidents and accidents related to pressure equipment have been duly collected for a number of years by the local inspectors and provided to the research team. The collected reports include basic information about type of event, involved equipment and materials, type and amount of loss. At the end there is a text describing more in detail damages, investigations and assumed causes. The texts are of different lengths, from a few lines up to five pages and more. They may include also photographs of the accident scene and evidences of metallographic tests. The number and type of pressure equipment in service in the competence district of each participating inspectors is known with a low uncertainty degree, because the obligations to authorities by the plant operators.

Phase 2 Basic Statistic In this phase the goal was providing parameters, namely FRs and FMs, trustable for risk based decisions and management. The calculation of FRs is quite simple and has been faced using trivial statistical methods. The FMs are more challenging and results obtained by trivial methods may be unsatisfactory. As a large diversity of equipment is involved, with many types of fault; the statistical analysis of the documents had to balance two conflicting needs: the different classes of membership (e.g. types of activities, types of equipment, types of damage, class of age) should be discriminated to have interesting and detailed results; but the 
sample cannot be fragmented too much, to avoid a loss of statistical significance. This problems hinder also a profitable use of advanced methods such as Bayesian belief network. Phase 3 Semantic phase. The goal is to distil as much knowledge as possible from the collected reports. The idea was to assume the consistency between report stories and actual events, so to measure the "distance" (or better the vicinity) between events through the "semantic distance" between reports. According to the MinHash algorithm proximity is defined as:

$\mathrm{P}_{\mathrm{a}-\mathrm{b}}=\left(\frac{\mathrm{K}^{\mathrm{a}} \cap \mathrm{K}^{\mathrm{b}}}{\mathrm{K}^{\mathrm{a}} \cup \mathrm{K}^{\mathrm{b}}}\right)$

where pa-b = proximity of event records $a$ and $b$; $\mathrm{Ka}$ and $\mathrm{Kb}$ key sentences singled out respectively in the event records $a$ and $b$ by the search engine.

In order to apply the proximity index, automated summaries are essential. For trustable summaries the possible keywords should be organized a priori. Synonyms should be duly considered. Furthermore the taxonomy of equipment, parts, industries, degradation mechanisms, processes, materials, damages and consequences are essential to discriminate general words (representing higher level classes) and detail words representing lower level in the taxonomies. The organization of the taxonomies of the equipment under pressure in relation to safety was developed a time ago by Ansaldi \& al. [2] and recently updated by Bragatto \& al. [3]. The set of possible key words may be considered as a n-dimensional space where each event may be placed. Proximity index define a metric for this space, thus it is possible to build a set of similar events, which may be considered "frequent failures". A cluster of failures is eligible as "frequent failure" if the number of events is $>3$ and all proximity are higher than 0.67 . The minimum of the proximity index is defined "radius of the cluster". The tool used in phase 3 is IBM Omnifind $\odot$, which provides: advanced search, multi language and semantic distance. Automated summaries are also produced [13]. Using the strength of advanced search, a number of clusters may be obtained, which may be considered typical or frequent cases.

\section{Phase 4 Extension}

In the process industries, the need to share information on incidents to learn from past mistakes and improve the future has been recognized for decades. To the first times most information was proprietary, but at now there are a number of national and international authoritative sources of information on accidents in the web, open to the public access. The reports included in those sources are focused on accidents happened in different industries. As many accidents in a few industries (e.g. oil industry) are caused by a failure in pressure equipment, the accidents' databases are a valuable source of free information on failure modes of pressure equipment. In the present paper the following websites have been considered: Barpi/Aria a general accident database for all industries in French language [9]; eMars a database on major accidents in chemical industry, in English language [10]; INAIL/Informo an occupational injuries database in Italian language [14]. A further source included was CCPS/Beacon, which provide, for teaching purpose, the description, in different languages, of a number of accidents [08]. The sources named above were extracted a small number of sheets, which were supposed relevant with the equipment failures. The capabilities of Omnifind(C) has been stressed to overcome the language differences as well as even the inconsistencies and fragmentation of those sources.

\section{RESULTS Statistics}

The failure rates are accordance with International bodies. Boilers have been discriminated by other types of equipment as the frequency is much higher but consequences much lower. FRs are reported in Table 1. Rates are in accordance with FRED [12] and API [01] official data, but for steam generators.

Table 1: Failure rates

\begin{tabular}{|l|r||r|c||}
\hline & $\begin{array}{c}\text { minor / } \\
\text { repairable }\end{array}$ & $\begin{array}{c}\text { non } \\
\text { repairable }\end{array}$ & major \\
\hline Other Emilia & $6.6 \mathrm{E}-05$ & $3.8 \mathrm{E}-05$ & $6.9 \mathrm{E}-06$ \\
\hline Steam Emilia & $4.7 \mathrm{E}-04$ & $2.5 \mathrm{E}-04$ & $3.1 \mathrm{E}-05$ \\
\hline Other Varese & $8.9 \mathrm{E}-05$ & $5.4 \mathrm{E}-04$ & $1.8 \mathrm{E}-05$ \\
\hline Steam Varese & $9.0 \mathrm{E}-03$ & $1.3 \mathrm{E}-03$ & - \\
\hline
\end{tabular}

An integrated and very general picture of the failure modes is shown in table 2. The prevalence of failure to the steam generators is also due to the industries prevailing in the two territories (textiles and food). Of course the prevailing types of equipment determine the type of failed part. Under the name "cracks" are included multiple cracks, spread cracks, cracks through and holes, whilst a single crack was not considered a real failure. 
Regarding the age, equipment with less than 3 years has been considered new, within 3 and 30 years medium, within 30 and 45 years mature, more than 40 obsolete. The assumed causes have been clustered in three generic types of physical causes and three procedural causes. The cause in Table 2 are intentionally naïve, as the understanding of the actual or root causes needs a deeper discussion. The investigation about causes depend on the type of damage mechanism, which, in turn, depend on the type of process and on the type of industry. An attempt of understanding of the cause has been made by using a Bayesian Network, which has provided a number of probabilistic relations between industry, type of equipment, part, damage and causes. Something may be found in Table 3 and 4 , where the percent probability of each major cause for each type of major failure is plotted. Less frequent causes are not included. Table 3 refers just to process industries, whilst table 4 refers to manufacturing industries. The greatest difficulty is the diversity of events and the limited number of comparable cases, which spread too much the uncertainties of the results.

\section{Frequent failures}

The essential results of semantic search are shown in Table 5. The key sentences describing the typical or "frequent failure" are shown in the first column. for each frequent failure shows a photo shot as taken by inspectors on the spot investigation. On the right column of the table the number of similar sentences in the cluster and the minimum proximity radius of the cluster, as defined in eq. 1.

Table 2: Failure modes

\begin{tabular}{|c|c|c|c|c|c|c|c|}
\hline \multicolumn{2}{|l|}{ a) Industry } & \multicolumn{2}{|c|}{ b) Type of equipment } & \multicolumn{2}{|c|}{ c) Affected part } & \multicolumn{2}{|c|}{ d) Type of damage } \\
\hline Process & $33 \%$ & Steam gen. & $56 \%$ & $\begin{array}{l}\text { Tube - sheet } \\
\text {-bundle }\end{array}$ & $38 \%$ & Cracks & $58 \%$ \\
\hline Manufacturing & $25 \%$ & Tank & $14 \%$ & Shell \& End & $28 \%$ & Structural & $15 \%$ \\
\hline Health & $22 \%$ & Column & $7 \%$ & Full & $12 \%$ & Blast \&fire & $14 \%$ \\
\hline Trans & $9 \%$ & Autoclave & $6 \%$ & $\begin{array}{l}\text { Nozzles \& } \\
\text { Manifold }\end{array}$ & $10 \%$ & Damaged & $9 \%$ \\
\hline Waste & $7 \%$ & Cylinder & $5 \%$ & Firebox & $5 \%$ & Occlusion & $2 \%$ \\
\hline \multirow[t]{4}{*}{ Other } & $5 \%$ & Reactors & $3 \%$ & Opening & $5 \%$ & \multirow[t]{4}{*}{ Fouling } & $1 \%$ \\
\hline & & Piping & $3 \%$ & Valve & $2 \%$ & & \\
\hline & & Furnace & $2 \%$ & \multirow[t]{2}{*}{ Other } & \multirow[t]{2}{*}{$2 \%$} & & \\
\hline & & Exchanger & $2 \%$ & & & & \\
\hline \multicolumn{4}{|l|}{ e) Class of age } & \multicolumn{4}{|c|}{ f) Assumed cause } \\
\hline \multicolumn{3}{|l|}{ New } & $6 \%$ & \multicolumn{3}{|l|}{ Corrosion } & $39 \%$ \\
\hline \multicolumn{3}{|l|}{ Medium } & $69 \%$ & \multicolumn{3}{|l|}{ Thermal stress } & $6 \%$ \\
\hline \multicolumn{3}{|l|}{ Mature } & $22 \%$ & \multicolumn{3}{|l|}{ Mechanical } & $5 \%$ \\
\hline \multicolumn{3}{|l|}{ Obsolete } & $3 \%$ & \multicolumn{3}{|c|}{ Operation control } & $26 \%$ \\
\hline \multirow{2}{*}{\multicolumn{4}{|c|}{ 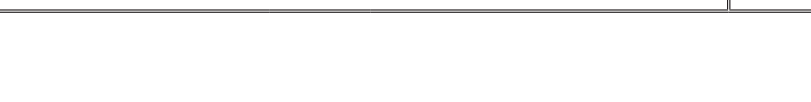 }} & \multicolumn{3}{|l|}{ Construction } & $14 \%$ \\
\hline & & & & \multicolumn{3}{|l|}{ Design } & $10 \%$ \\
\hline
\end{tabular}

Table 3: The causes for process industries

\begin{tabular}{|l|c|c|c|c||c|c|}
\hline \multicolumn{7}{|c|}{ Types of Damage } \\
\hline Root Causes & Crack Thru & Deformed & Multi Crack & Rupture & Peeling & Blast \\
\hline \hline Improper Operation & 13.2 & 10.9 & 13.9 & 10.6 & 5.5 & 11.2 \\
\hline Inadeqaute Design & 10.2 & 13.2 & 9.0 & 9.4 & 7.0 & 11.2 \\
\hline Improper Welding & 15.3 & - & 20.9 & 8.5 & - & 10.1 \\
\hline Inadeqaute Material & 9.0 & - & 10.6 & - & 14.1 & - \\
\hline
\end{tabular}

Table 4: The causes for manufacturing industries

\begin{tabular}{|l|c|c|c|c|c|c|}
\hline \multicolumn{9}{|c|}{ Types of Damage } \\
\hline Root Causes & Crack Thru & Deformed & Multi Crack & Rupture & Peeling & Blast \\
\hline Improper Operation & 15.3 & 22.4 & 15.0 & 10.0 & - & 32.4 \\
\hline Inadeqaute Design & 8.7 & 15.6 & 9.6 & 10.0 & - & 12.6 \\
\hline Improper Welding & 15.5 & - & 27.4 & 7.3 & 14.9 & 9.5 \\
\hline Inadeqaute Material & 9.0 & - & 10.6 & - & - & - \\
\hline \hline
\end{tabular}


Table 5: Major "frequent failures

\begin{tabular}{|c|c|c|}
\hline & Frequent failures & $\begin{array}{l}\text { Number of items/ } \\
\text { Proximity radius }\end{array}$ \\
\hline & $\begin{array}{l}\text { Deformation for Thermal Stress. } \\
\text { Steam boilers in the textile or food indu-stry: plastic defor-mation of parts } \\
\text { (tube plate, tube shell, furnace) due to excessive thermal load (e.g. fault of } \\
\text { water supply) }\end{array}$ & $4 / 91 \%$ \\
\hline & $\begin{array}{l}\text { External Corrosion of buried tanks. } \\
\text { Shell and bottoms of buried tanks may be affected by fast corrosion and } \\
\text { peeling due to ingress of water it inside the trench. Consequnces include gas } \\
\text { leakage and accdents. }\end{array}$ & $3 / 82 \%$ \\
\hline & $\begin{array}{l}\text { Reactor and Aggresive Chemicals. } \\
\text { Batch reactors in the chemical industry. Dete-rioration and breakage of parts } \\
\text { - stress corrosion due to the use of aggressive substances (e.g. chlorides) }\end{array}$ & $3 / 80 \%$ \\
\hline & $\begin{array}{l}\text { Corrosion Under Insulation. } \\
\text { In Heat Exchanger, and reactors, a little leakage cause an accumulation of } \\
\text { fluid under insulation, which remain undetected for a long time until cause } \\
\text { major damage. }\end{array}$ & $3 / 79 \%$ \\
\hline & $\begin{array}{l}\text { Fouling \& overheating. } \\
\text { Rust and sediment accumulated on the tube during service reduce heat ex- } \\
\text { change capacity. In reactors uncontrolled temperature may lead to runaway } \\
\text { phenomena with severe consequences }\end{array}$ & $3 / 76 \%$ \\
\hline & $\begin{array}{l}\text { Inappropriate Pressure Tests. } \\
\text { In tanks and in cylinders "Hydraulic or Pneumatic Tests" made without com- } \\
\text { plying the good practices causes catastrophic blasts of vessels with possibly } \\
\text { with severe consequence. }\end{array}$ & $3 / 73 \%$ \\
\hline & $\begin{array}{l}\text { Untreated Water into boilers. } \\
\text { Steam boilers, autoclaves or heat exchangers. Corrosion and fouling spread } \\
\text { over various parts (shell and tube, tube sheet, valves) due to water treatment } \\
\text { inadequate or absent. }\end{array}$ & $6 / 73 \%$ \\
\hline $\begin{array}{llll} & 5 & & \end{array}$ & $\begin{array}{l}\text { Liquid Hammer. } \\
\text { In heat exchangers, steam generators and other types of equipment recurrent } \\
\text { "liquid hammers" on tubes cause fatigue and ruptures possibly with severe } \\
\text { consequence. }\end{array}$ & $3 / 71 \%$ \\
\hline & $\begin{array}{l}\text { Switching Fuels. } \\
\text { In steam boilers the change of fuels or the use of unconventional fuels } \\
\text { causes thermal stresses and cracks. }\end{array}$ & $3 / 70 \%$ \\
\hline
\end{tabular}

It has to be stressed that the searches that have produce the "frequent failure" summarized in table 5 have involved some 120 sheets coming from inspectors on the field and some 30 sheets picked from open access sources. Although this is a limited sample, the fact that certain faults will be repeated three or more times, it leads to trust that they are actually "frequent". There is no room here to discuss in detail each "frequent failure" in table 5. Some of these are known problems, for which recommended good practices could be found in the technical literature, including Corrosion Under Insulation, Liquid Hammers. Unfortunately practitioners forget quickly and after a time past mistakes return. Other results are less obvi- ous. For example accidents caused by pressure tests are worrying. Hydraulic and pneumatic tests are dangerous; if they cannot be avoided must be entrusted to aware and skilled persons. In previous paragraphs the FRs of steam generators have been discussed, as much higher than other equipment. A couple of frequent failures, including untreated water and switching fuels, highlight serious problems in the management of the boilers. These incidents were recorded in the textiles, food, waste and health sectors, where at the time of the events management systems were not yet present. More frequent inspections and accurate, conducted within a safety management system, are able to avoid many of the frequent failures, as well as accidents deriving from them. 


\section{CONCLUSIONS}

The efforts made by generations of scholars who came before us to define a very solid base of knowledge on failure modes and rate of pressure equipment are impossible to this day, as based on the "command and control" approach, where every technical issues were directly controlled by one national authority. Now technical duties are spread to many public and private bodies, independent each other. The increasing number of data on failures, accidents and incidents, which are becoming available in the space of the public domain, and the power of semantic search, as demonstrated by this paper, are the only possible alternative for reviving and updating the knowledge on pressure equipment failure and achieve a level again comparable to that described by the article by S. Bush, cited in the introduction. Obviously there are many problems that in the past were not present. In particular, the data are completely different, fragmented and disaggregated. The different languages and even jargons contribute to the difficulties. Also the level of detail is extremely different. According to various sources there are very precise details on materials and processes, or only feedback of organizational management. In some cases the reports are that have been reported within modules, which obviously must not be included in the search. It is precisely the strength of the semantic search engine that can help, but obviously they must be used by experts who know very well the rules that underlay the matter. In the present experiments just a few sheets have been retrieved from open access database and processed with the proprietary data. As the purpose of the experiment was just explorative, that is adequate, but for a larger campaign the issue of synchronizing with different open access accident databases should be faced and it is not easy at all. A even major obstacle to the development of a shared knowledge on pressure equipment safety is the ownership of data on failures. In some cases these data may have a competitive value and reasonable confidentiality, but in many cases the data can be purified and be shared to the benefit of all. It is therefore important that regulators encourage companies to publish the non-confidential data that may be significant for improving safety. In case of accident, with or without victims, institutions that can intervene are different from one district to another and, more importantly, from country to country. For this rea- son it would be useful define the requirements on the minimum information that must be collected and shared. Something like that already exists at EU level for reporting serious accidents in Seveso establishments and could inspire a very simplified model, suitable for all industries.

\section{REFERENCES}

1) API RP 581, Risk-Based Inspection Technology 2nd edition 2008, Washington

2) Ansaldi, S., Bragatto, P., Camossi, E., Giannini, F., Monti, M., Pittiglio, P. A knowledgebased tool for risk prevention on pressure equipments (2006) Computer-Aided Design and Applications, 3 (1-4), pp. 99-108

3) Bragatto P. Ansaldi S. Delle Site C. A., 2013, Pooled Knowledge Basis on Pressure Equipment Failures to Improve Risk Management in Italy Chemical Engineering Transactions, 33, 433-438

4) Bragatto, P., Delle Site, C., Faragnoli, A. 2012 Opportunities and threats of risk based inspections: The new Italian legislation on pressure equipment inspection Chemical Engineering Transactions, 26, pp. 177-182

5) Bush, S.H. 1988, Statistic of Pressure Vessel and Piping Failures. J. of Pressure Vessel Technology; 110: 225-233

6) Manuel, H.J. Kooi, E.S. Bellamy, L.J. Mud, M.L. Oh, J.I.H. 2012 De-riving major accident failure frequencies with a story builder analysis of reportable accidents. Process Safety Progress; 31(4):381-389

7) Pittiglio, P. Bragatto, P. Delle Site, C. 2014 Updated failure rates and risk management in process industries Energy Procedia 45: 1364 -1371

8) www.aiche.org/ccps/resources/processsafety-beacon/

9) www.aria.developpement-durable.gouv.fr/ in French

10) https://emars.jrc.ec.europa.eu/

11) http://salute.regione.emilia-romagna.it/documentazione/rapporti/anomalie_apparecchi_ a_pressione.pdf in Italian

12) www.hse.gov.uk/landuseplanning/failurerates.pdf

13) www-01.ibm.com/software/ecm/omnifind/

14) www.ispesl.it/getinf/informo/ in Italian

Paper sent to revision: 21.11.2015.

Paper ready for publication: 11.12.2015. 